\title{
The Application of Neural Network and Logistic Regression in the Opera- tion Performance Warning of Power Grid
}

\author{
Yixin Sun ${ }^{1,2, *}$ XiaobaoYu ${ }^{1}$, QingyouYan ${ }^{1}$ and Zhongfu Tan ${ }^{1}$ \\ ${ }^{I}$ School of Economics and Management, North China Electric Power University, Beijing 102206, P.R. China \\ ${ }^{2}$ State Grid Energy Research Institute, Beijing 102209, P.R. China
}

\begin{abstract}
In this paper, for a given initially analyses the operation performance of grid enterprises, we divide index factors affecting operation performance of grid enterprise into two types: investment index and result index. For the the method of warning analysis, we establish models of neural network and logistics regression, using Matlab mathematical software to calculate and analyse the above-mentioned two types of indexes, concludes their relationship of correlation coefficient and regression coefficient. For scenarios analysis, we use models with 3 scenarios to study on operation performance risks of grid enterprises herein. Eventually we analyses actual data from various places empirically and arrives at the related conclusion. For conclusion, we hold the opinion that risks of operation performance may occur in the future if present investment cannot satisfy requirement for power grid development of various regions; increasing investment ratio may be likely to decrease occurrence rate of risks of power grid operation performance.
\end{abstract}

Keywords: Logistic regression, multi-scenario analysis, neural network, operation performance, warning.

\section{INTRODUCTION}

Currently many achievements have been made in research of power grid operation performance at home and abroad, while there exists a gap in the research on the warning of operation performance of the gird enterprise. Hence the paper revolves around further investigation of operation performance risks of the grid enterprise.

In terms of the research on operation performance of grid enterprises, domestic scholars like Lin Liqiong [1] provided an efficient and accurate warning assessment method and process by dividing power grid operation into four systems of power grid structure, power grid operation, equipment operation and maintenance and security management; Yan Zhixiong [2] raises an analysis method of power grid in view of DEA on the basis of analysing issues of present research situation of grid enterprises operation performance and considering characteristics of power grid operation, requirements of performance analysis and actual data comprehensively; the scholars including Xiao Qishi [3] have established index system of risk management of grid enterprises operation to monitor and analyse operation risks of grid enterprises and put a new model for enterprises risk management and research, building a model of risk management system relating to grid enterprise business process; the scholars including Chen Anwei $[4,5]$ have dissolved the investment into smart power grid by department in an efficient manner, applied input-output economy and various industrial sectors motivated by investment, and model and analysed influence on the growth of national investigated coordinated development regarding safety, reliability, progressiveness and interactivity of smart power grid; other domestic scholars have conducted relevant research on grid enterprise operation performance from their familiar research areas as well [6-10].

Compared with domestic research on the operation performance of the grid enterprise, foreign scholars have different research themes in this aspect; Litvinov Eugene [11] studies and analyses possible risks caused by power grid operation considering uncertainty generating from power market reform; Zhang Jinhui [12] analyses factors affecting the operation risks of power grid with the method of AHP and gives a fuzzy comprehensive evaluation on power grid operation; other foreign scholars propose different viewpoints on the operation risks of power grid from their own research angles $[13,14]$.

The paper uses mixing method of neural network [15, $16]$ and logistic regression $[17,18]$ to investigate warning of operation performance of power grid. Firstly, listing relevant indexes of the operation performance of power grid, selecting key indexes and sorting them into two types: result index and investment index; secondly, building correlation coefficient between investment index and result index with the method of neural network and building regression coefficient between investment index and result index with the method of logistic regression to establish relationship of the two types of indexes; thirdly, setting investment index values under different scenarios and respectively calculating result index values in the method of correlation coefficient and regression coefficient; finally, assessing power grid. operation perform- 
ance in the future under different scenarios with result index values and proposing pertinent suggestions.

\section{WARNING INDEX SYSTEM OF POWER GRID OPERATION PERFORMANCE}

The monitoring of grid enterprise operation [19] largely depends on index to reflect its business changes, efficiency and effectiveness and relevant indexes cover aspects of human resource, financial resource, material resource, power grid planning, infrastructure, operation, recondition and marketing. In such a huge index group, it is needed to establish a comprehensive assessment index system which could not only be used to assess grid enterprise operation overall but also analyse internal investment factors for the operation and development of the grid enterprise.

\subsection{Design Principle of Index System}

Building the index system of operation monitoring is conductive to evaluate grid enterprise operation performance overall which is required as followed in terms of index selection: Firstly, representativeness to reflect enterprise' operation fundamentals; Secondly, accuracy to reflect key evaluated operation factors accurately; Thirdly, features of being obtainable and measurable that are fixed and selected from common used indexes.

\subsection{Core Index of Operation Performance Warning}

With reference to basic ideas of Balance Score Card, the system is readjusted appropriately from four angles and after this it contains two types: investment-type and result-type. Among them investment index states basic conditions for enterprise operation, basic resource investment conditions and more; result index states final value of enterprise operation, including financial performance, energy-saving and emission-reduction performance and so on. Eight indexes are selected respectively and then divided into two key index groups in line with different requirements: Result index group and Investment index group, and go to Table 1 for index element details:

\section{SUMMARY OF WARNING MODEL}

\subsection{Design Thought of Model}

Warning model of grid enterprise operation performance is composed of three main sections: computational model of correlation coefficient between investment index and result index, computational model of regression coefficient and computational model of mixing warning. Computational model of correlation coefficient uses neural network model to establish neural network fitted model of investment index and result index and then calculate their correlation coefficient; computational model of regression coefficient uses the model of logistic regression to establish the regression coefficient fitted model of investment index and result index and then calculate their regression coefficient; computational model of mixing warning sets different situations, predict investment index, calculate result index value with different coefficients under different situations and conducts mixing analysis on the former calculated result values, thus obtaining the index that is most likely to threat power grid. The design flowchart of warning model is shown in Fig. (1).

\subsection{Computational Model of Correlation Coefficient}

In the paper a neural network model of optimum genetic algorithm is adopted to calculate correlation coefficient of investment index and result index. A neural network model of optimum genetic algorithm is made up of three sections: neural network structure identification, genetic algorithm optimization and neural network predication. The neural network structure identification inputs and outputs numbers of parameter in accordance with fitting function to identify neural network structure, and further ascertain individual length of genetic algorithm. In genetic algorithm optimization, the generic algorithm is used to optimize weights and thresholds of neural network so that each individual in the population has a weight and threshold of network ownership, each individual calculates individual fitness value through fitness function and then genetic algorithm could find the optimum fitness value to match an individual through choosing, crossover and variation operation. Neural network prediction makes use of genetic algorithm to get optimum indi-

Table 1. Investment index group and result index group.

\begin{tabular}{|c|c|c|c|}
\hline \multicolumn{2}{|c|}{ Investment Index Group } & No & Index \\
\hline \hline No & Index & 1 & Asset - liability ratio \\
\hline 1 & Total assets & 2 & Power supply stability \\
\hline 2 & Power & 3 & Electricity sales \\
\hline 3 & Gross installed capacity & 4 & Line loss rate \\
\hline 4 & Investment of capital construction & 5 & Total profit \\
\hline 5 & Technology improvement investment & 6 & Productivity of labour \\
\hline 6 & Marketing input & 7 & Load of power supply increase from investment in unit power grid \\
\hline 7 & Training input & 8 & Electricity sales increase from investment in unit power grid \\
\hline 8 & Informationization input & & \\
\hline
\end{tabular}




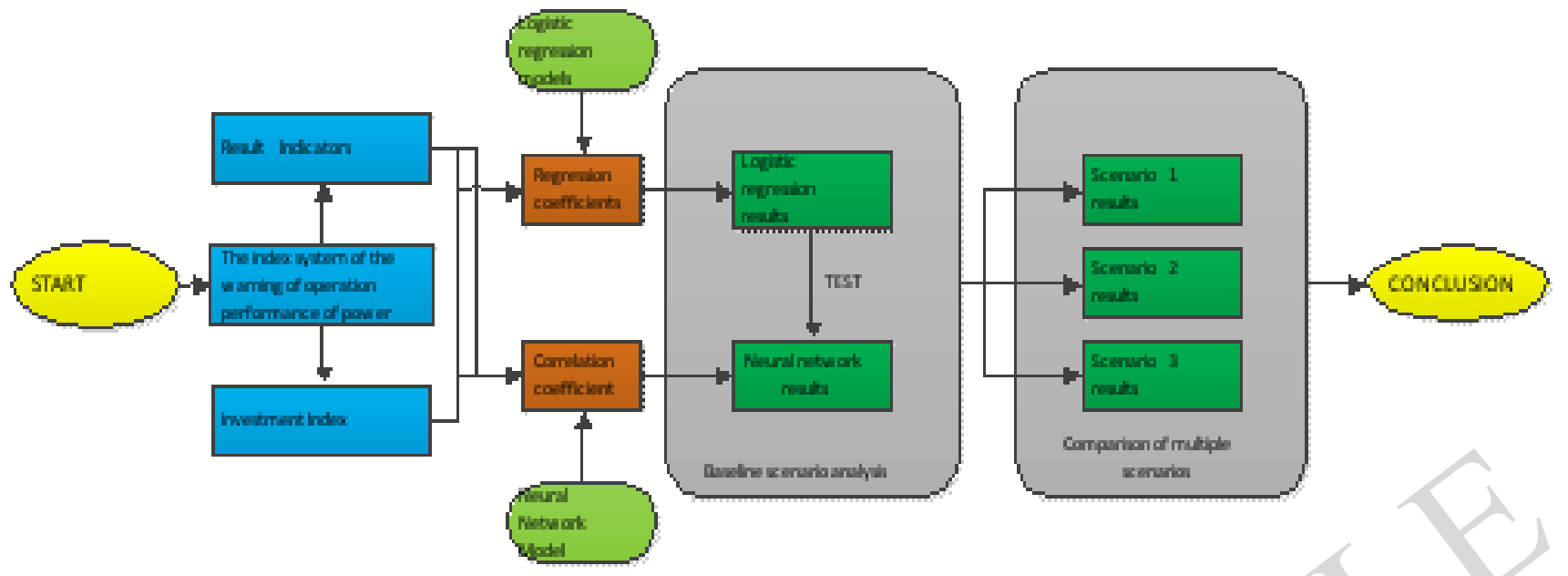

Fig. (1). Design flowchart of warning model.

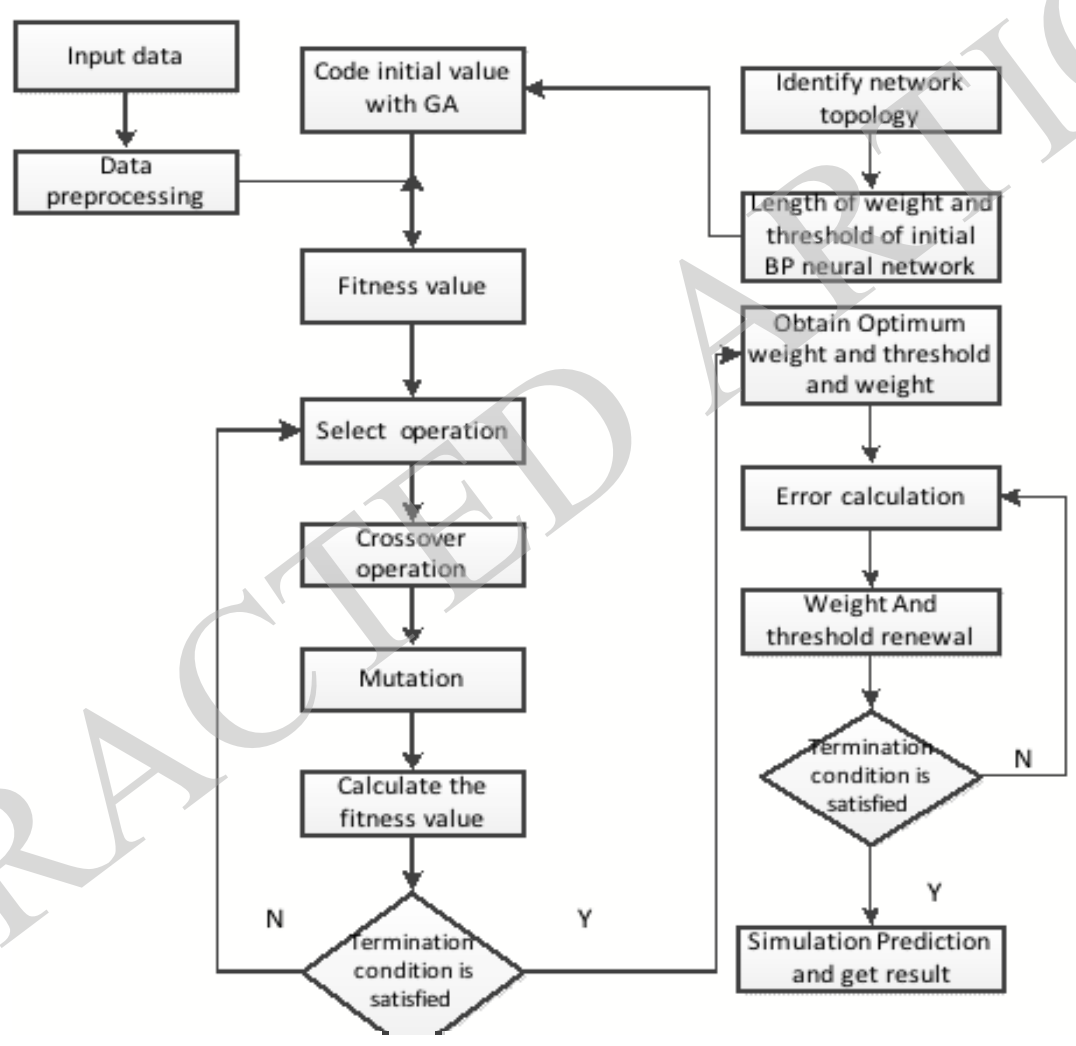

Fig. (2). Algorithm flowchart of generic algorithm.

vidual, assigns weight and thresholds initiations and outputs the function that is predicted after network practice. Algorithm flowchart of neural network.

Generic algorithm optimum neural network uses generic algorithm to optimize weight and threshold initiations so as to better predict the output of functions. The essential factors of generic algorithm optimum neural network are population initiation, fitness function, choice operation, crossover operation and variation operation.

To improve the accuracy of correlation coefficient calculation, the paper adopts many-to-one fitness model to select eight investment indexes as input index and one result index as output index and set hidden layer with 5. Fig. (3) for network design:

Calculation principle of neural network model is as follows:

$$
\begin{aligned}
& o_{k}=\psi\left(\sum_{i=1}^{q} w_{k i} \phi\left(\sum_{j=1}^{M} w_{i j} x_{j}+\theta_{i}\right)+a_{k}\right) \\
& \Delta \omega_{k i}=\eta \sum_{p=1}^{P} \sum_{k=1}^{L}\left(T_{k}^{p}-o_{k}^{p}\right) \cdot \psi^{\prime}\left(\text { net }_{k}\right) \bullet y_{i} \\
& \Delta \alpha_{k}=\eta \sum_{p=1}^{P} \sum_{k=1}^{L}\left(T_{k}^{p}-O_{k}^{p}\right) \cdot \psi^{\prime}\left(\text { net }_{k}\right)
\end{aligned}
$$




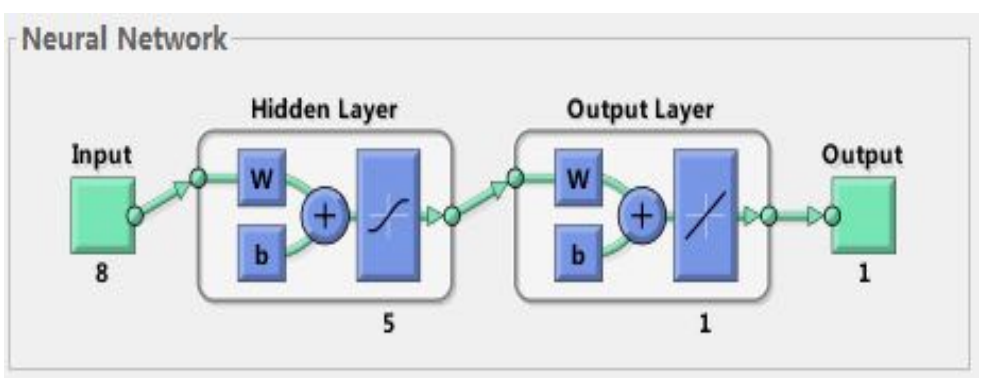

Fig. (3). diagram of neural network model.

$$
\begin{aligned}
& \Delta w_{i j}=\eta \sum_{p=1}^{P} \sum_{k=1}^{L}\left(T_{k}^{p}-\boldsymbol{O}_{k}^{p}\right) \cdot \psi^{\prime}\left(\text { net }_{k}\right) \cdot y_{i} \bullet w_{k i} \bullet \phi\left(\text { net }_{i}\right) \cdot x_{j} \\
& \Delta \theta_{i}=\eta \sum_{p=1}^{P} \sum_{k=1}^{L}\left(T_{k}^{p}-O_{k}^{p}\right) \cdot \psi^{\prime}\left(\text { net }_{k}\right) \bullet w_{k i} \bullet \phi\left(\text { net }_{i}\right)
\end{aligned}
$$

In formula,

$x_{j}$ represents an input layer of node $j, j=1, \ldots, M$;

$w_{i j}$ represents the weights between the hidden layer node $i$ to the input layer node $j$;

$\theta_{i}$ represents a threshold value of the hidden layer node $i$

$\phi(x)$ represents the hidden layer activation function;

$w_{k i}$ represents the weights between the hidden layer node $i$ to the output layer node $k, i=1, \ldots, q$;

$a_{k}$ represents a threshold value of the output layer node $k, k=1, \ldots, L$;

$\psi(x)$ represents the output layer activation function;

$O_{k}$ represents the output of the output layer node $k$;

$\mathrm{P}$ denotes the number of training samples;

$n t_{i}$ represents the input of the hidden layer node $i$;

$y_{i}$ represents the output of the hidden layer node $i$;

$n e t_{k}$ represents the input of the output layer node $k$;

$\Delta w_{k i}$ represents the correction amount of the output layer weights;

$\Delta a_{k}$ represents the correction amount output layer threshold value;

$\Delta w_{i j}$ represents the correction amount of the hidden layer weights;

$\Delta \theta_{i}$ represents the correction amount hidden layer threshold value.

It is needed to input investment index and result index data in the past ten years, run the programmed model and compute correlation coefficients of input layer and hidden layer, and correlation coefficients of hidden layer and output layer to calculate correlation coefficients of investment index and result index.

\subsection{Computational Model of Regression Coefficient}

In this paper Logarithmic function coefficient model is adopted for dimensionless processing of the data.

$d_{i}=\frac{\ln \left(x_{i}\right)-\ln \left(X_{0}\right)}{\ln \left(X_{1}\right)-\ln \left(X_{0}\right)} \times 40+60$

Logistic regression algorithm is employed to calculate regression coefficient of investment index and result index. Occurrence probability of an incident with values ranging from 0 to 1 , and $1-p$ is the non-occurrence probability of the incident. The natural $\log$ graph of the ratio of $p /(1-p)$ could be obtained and record LogitP with values of LogitP ranging from negative infinity to positive infinity. Frame liner regression equation and LogitP is a dependent variable:

$\log i t P=a+b_{1} x_{1}+b_{2} x_{2}+\ldots+b_{m} x_{m}$

And thus get:

$P=\frac{1}{1+\exp \left(a+b_{1} x_{1}+b_{2} x_{2}+\ldots+b_{m} x_{m}\right)}$

$1-P=\frac{\exp \left(a+b_{1} x_{1}+b_{2} x_{2}+\ldots+b_{m} x_{m}\right)}{1+\exp \left(a+b_{1} x_{1}+b_{2} x_{2}+\ldots+b_{m} x_{m}\right)}$

Errors of logistic regression are subjected to binomial distribution instead of normal distribution and estimate parameters with maximum likelihood classifier in fitness. In the model $a$ is a constant term and it will the natural log graph of a ratio (ratio of $Y=1$ and $Y=0$ ) when the variable is 0 , and $b_{i}$ is logistic regression coefficient and it means increase by one unit of this independent variable will cause the change of natural log graph of a ratio when other this independent variables are fixed.

\subsection{Multi-scenario Warning Models}

The correlation coefficient and regression coefficient of two types of indexes can be obtained through building models of neural network and logistic regression and combing index data. Setting different investment scenarios, computing investment index value under different investment scenarios and calculating result index values under scenarios through combing correlation coefficient and regression coefficient. The threshold value could be fixed with reference to related regulations and expert suggestions. 
A comparative analysis of result investment value and thresholds value is needed to discover hidden dangerous indexes so as to conduct warning analysis under scenarios of different investment based on analytic results.

\section{EMPIRICAL ANALYSIS}

Data of investment index and result index from 25 provinces in 2012 is selected and initially applied to dimensionless method. Then the correlation coefficient and regression coefficient of investment index and result index are respectively calculated with models of neural network and logistic regression on the basis of non-dimensionalized data.

\subsection{Baseline Scenario Analysis}

It is required to build neural network model of correlation coefficient calculation, take eight investment indexes as input layer data and eight result indexes as output data and compute with toolbox of the program of matlab. The correlation coefficient of investment index and result index could be derived from relationships of weights coefficient bet, as shown in Table 2.

Three investment scenarios are set and distribution of grid enterprise operation performance in different provinces under each scenario is analysed.

It is proved that result index value calculated through neural network model can be tested by logistic regression compared with Fig (4). Based on the number of 0.5 , if result index value in more than 0.5 , the value is 1 and otherwise the value is 0 . The fact that the numbers of results coinciding with logistic regression is 143 and accuracy rate is $71.5 \%$ shows correlation coefficients of two types of indexes computed through neural network model are basi- cally rational.

Distribution of result index values in various provinces in the year of 2015 under baseline scenario can be pictured with assistance of Table 3 and it is showed in Fig. (4). In accordance with the basic principles and calculation formulas to deal with data herein, the standard index value is 0.6 which means thresholds value of the risk of result index is set as 0.6 .

From Fig. (4) it is evidently to see provinces with potential risks. From Table 4, under scenario 1, various places are likely to face risks in the future and practically more will occur in regions with slowing economies. For instance in Qinghai, other indexes are likely to indicate risks except that of increase investment in unit grid; in Jiangsu province, other indexes are unlikely to indicate risks except that line loss rate and electricity selling increase in unit grip are probably cause risks. It is very hard for the current investment to avoid hidden risks and more investment is required to improve investment ratio, thus improving result indexes and reducing the occurrence probability of risks.

\subsection{Multi-scenario Analysis}

Set the parameters of different scenarios according to Table 2, for instance in Beijing, the result indexes of Beijing in Scenario 2 and Scenario 3 could be calculated with parameters set in different scenarios and they are showed in Table 3.

From Table $\mathbf{3}$ and Fig. (5), indexes are evidently enhanced. Taking line loss rate as an example, in scenario 1, the line loss rate in Beijing is in risk areas, and it is in safety areas in scenario 2 and 3. This demonstrate that increasing investment ratio has definite effect on enhancing result index value and is conductive to avoid power network operation

Table 2. Details of scenario parameters.

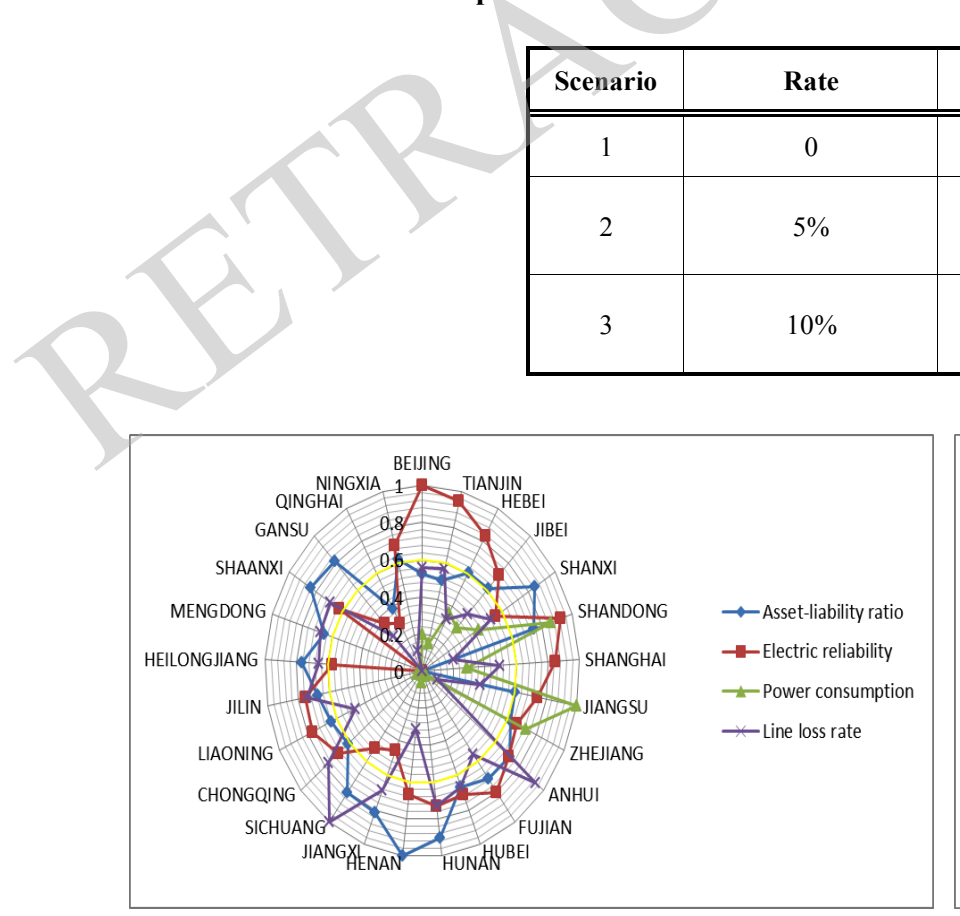

\begin{tabular}{|c|}
\hline Remark \\
\hline Historical trend fitted value \\
$\begin{array}{c}\text { Increase by } 5 \% \text { based on fitted value } \\
\text { of historical trend }\end{array}$ \\
$\begin{array}{c}\text { Increase by } 10 \% \text { based on fitted } \\
\text { value of historical trend }\end{array}$ \\
\hline
\end{tabular}

Fig. (4). Distribution of Result index values of provinces.

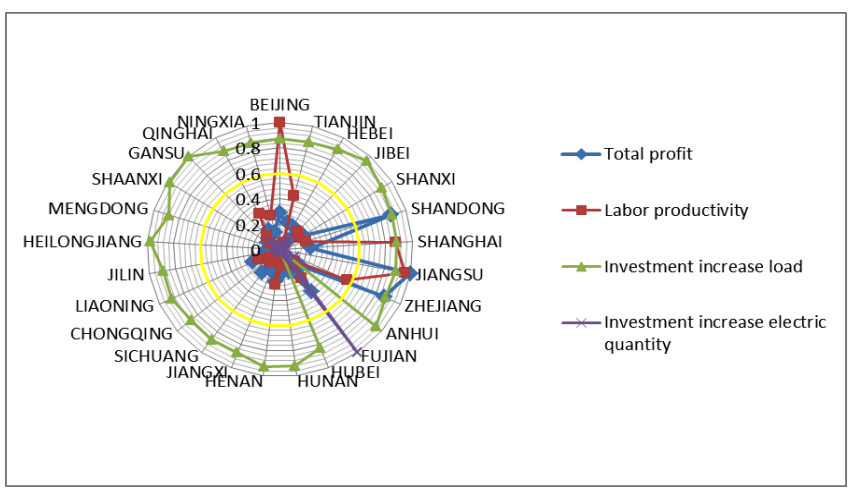


Table 3. Result index in areas of beijing under different scenarios.

\begin{tabular}{|c|c|c|c|c|c|c|c|c|}
\hline Scenario & $\begin{array}{c}\text { Asset- } \\
\text { liability ratio }\end{array}$ & $\begin{array}{l}\text { Electric } \\
\text { reliability }\end{array}$ & $\begin{array}{l}\text { Power con- } \\
\text { sumption }\end{array}$ & Line loss rate & Total profit & $\begin{array}{l}\text { Labor pro- } \\
\text { ductivity }\end{array}$ & $\begin{array}{c}\text { Investment } \\
\text { increase load }\end{array}$ & $\begin{array}{c}\text { Investment } \\
\text { increase } \\
\text { electric } \\
\text { quantity }\end{array}$ \\
\hline Scenario 1 & 0.524726 & 0.999999 & 0.202052 & 0.556985 & 0.291727 & 0.999999 & 0.873368 & 0.034647 \\
\hline Scenario 2 & 0.583982 & 0.999999 & 0.435678 & 0.603421 & 0.342571 & 0.999999 & 0.887463 & 0.234165 \\
\hline
\end{tabular}

Fig. (5). Distribution of result index in areas of beijing under different scenarios.

risks. From analytic study of areas in Beijing, it is concluded that some result index will have risks in various places in 2015 under current investment standard and increasing investment ration could reduce occurrence ratio of such risks but cannot completely avoid them. Therefore, relevant department should take other measures to avert risks in the future in addition to increasing investment ratio.

\section{CONCLUSION}

In the paper research into future power grid operation performance risks have been done through building indicator system of power grid operation performance which mainly contains two categories of indicators: investment indicator and result indicator, based on the analysis of the relationship between two indictors. Initially, computing correlation coefficient between two types of indicators through building the model of neural network; furthermore, computing regression coefficient between two types of indicators through building logistic regression model; afterwards, calculating and analysing risks for power grid operation performance in the future in various regions under bassline investment scenario; finally, calculating and analysing risks for power grid operation performance in the future in Beijing under bassline investment scenario.

(1) In the paper investment indicators and result indicators selected to predict risks for power grid operation performance in the future are reprensetative and fittign situation of correlation coefficeinent of neural network model shows distinct correlation relationship between two types of indica- tors. All ablove demonstrate that the research foudation is belivable

(2) Comparasion between values from regression coefficient computation and values from neural network model prediction demonstrates that accuracy rate of risk predication througn neural network model is up to $71.5 \%$, thus showing the predication results of future result index through neural network model are belivable

(3) Under baseline scenario power grid operaion performance risks are serious in various places in the year of 2015 and particullay in regions with slowing economies riskful result indexes are many. Measures should be taken in various places to decrease occurance rate of risk to avodi such situations.

(4) There comes the conclusion that from investigation of power grid operation performance under scenarios of different investment ratios increasing investment ration may be likely to decrease dangerous indexes in a certain but cannot insure against risks. It manifests that increasing investment may be considered as an efficient mean to minimize power grid operation risks without

In the paper increasing investment ratio means increasing all investment ratios of investment indexes and the purpose of this assumption is to analyze effects on risks reduction of the grid enterprise incurred by the increase of investment ratio. And matters on research of readjusting investment ratios of investment indexes to minimize risks to the greatest extent further are not discussed in the paper and will be made it in the future. 


\section{CONFLICT OF INTEREST}

The authors confirm that this article content has no conflict of interest.

\section{ACKNOWLEDGEMENT}

This paper belongs to the project of the "State Grid science and technology projects", No.kjxm20130401; State Grid Energy Research Institute.

\section{REFERENCES}

[1] L. Q. Lin, S. J. Chen, and Z. F. Tan, "Fuzzy Fault Tree Analysis Model for Grid Operation Risk Warning Evaluation”, East China Electric Power, vol. 2, pp. 229-235, 2014.

[2] Z. X. Yan, Analysis of Network Performance based on Data Mining. Hangzhou: Zhejiang University, 2014.

[3] Q. S. Xiao, W. Wu, and P. F. Zhang, "Operation Risk Management System Model and Control Mechanism in Power Grid Enterprise", East China Electric Power University, vol. 8, pp. 1691$1694,2014$.

[4] A. W. Chen, Research on the Technical Economic Problems of the Smart Grid. Chongqin: Chongqin University, 2012.

[5] P. Li, Smart Grid Operation Management Risk Element Transmission Model and Decision Support System Research. Beijing: North China Electric Power University, 2014.

[6] M. L. Wang, Research on the Model of the Company's Financial Early Warning Monitoring Mechanism and its Application Method. Dalian: Dalian University of Technology, 2006.

[7] S. K. Yang and Q. A. Chen, "Impact on the Provincial Power Grid Operation and the Countermeasures of the Establishment of Power Market", China Market, vol. 36, pp. 18-19, 2006.

[8] Y. Cai, Research and Application of Data Mining Technology in Grid Operation Monitoring Platform. Shanghai: Shanghai Jiao Tong University, 2013.
[9] Z. L. Liu, "Evaluation on developing level of unban agglomeration derived from resources exploration", Journal of Applied Sciences, vol. 13, no. 21, pp. 4702-4707, 2013.

[10] J. H. Shao, "Analysis on Risk Management of Power Grid Enterprise", Shanghai quality, vol. 12, pp. 54-57, 2010.

[11] T. X. Zheng and E. Litvinov, "Operational Risk Management in the Future Grid Operation”, in 2011 IEEE Power and Energy Society General Meeting, 2011.

[12] J. H. Zhang, "Assessment on operational risk in power grid enterprises based on variable weight fuzzy evaluation", in 2009 International Conference on Energy and Environment Technology Proceedings, 2009, vol 2, pp. 92-95.

[13] I. Valdir, C. Ernesto, and T. C. Jonas, "A Procedure for Operational Use of Wave Hindcasts to Identify Landfall of Heavy Swell", Weather and Forecasting, vol. 29, pp. 349-365, 2014.

[14] C. B. Li, "Analysis of wind power generation operation management risk in China”, Renewable Energy, vol. 64, pp. 266-275, 2014.

[15] S. X. Wang and N. Zhang, "Short-term Output Power Forecast of Photovoltaic based on a Grey and Neural Network Hybrid Model", Automation of electric power systems, vol. 19, pp. 37-41, 2012 .

[16] H. T. Shi, J. L. Yang, and M. S. Ding, "A short Term Wind Power Prediction Method based on Wavelet Decomposition and BP Neural Network", Automation of electric power systems, vol. 16, pp. 44-48, 2011.

[17] X. L. Zhang, "Research on Enterprise Financial Early-warning Positive Logic Regression Model", Search, vol. 1, pp. 36-38, 2012.

[18] L. Y. Liu, Research and Application of Recommendation Technology based on Logistic Regression. Xi-an: University of Electronic Science and Technology, 2013.

[19] Y. T. Wang, Q. Chen, Y. J. Liu, and Y. L. Xu, "The Design of Electric Power Enterprise Operation Monitoring (Control) Information System Application Architecture", Group technology and production modernization, vol. 1, pp. 32-38, 2013.

[20] J. Y. Yue and S. Y. Jin, "The Construction of Evaluation System based on the Balanced Scorecard", Heilongjiang foreign trade and economic cooperation, vol. 9, pp. 112-115, 2011.

Received: September 16, 2014

This is an open access article licensed under the terms of the (https://creativecommons.org/licenses/by/4.0/legalcode), which permits unrestricted, non-commercial use, distribution and reproduction in any medium, provided the work is properly cited. 